\title{
Food and agriculture restoration management involving networked groups (the FARMING network)
}

\author{
A. Nisbet and J. Mercer \\ National Radiological Protection Board, Environmental Assessments Department, \\ Chilton OX11 ORQ, U.K.
}

\begin{abstract}
Following a nuclear accident, stakeholder involvement is essential in the formulation of strategies for maintaining agricultural production and food safety. The importance of setting up national stakeholder groups as part of contingency planning has been illustrated by the success of the Agriculture and Food Countermeasures Working Group in the UK. In recognition of this, the European Commission is providing financial support to develop the FARMING network, initially involving stakeholder groups in the UK, Finland, France, Belgium and Greece. These national groups contain individuals involved in making policy decisions within government departments and agencies, the food and agriculture industries, as well as individuals with specialist expertise. The groups meet annually as part of pre-accident planning to debate and judge a range of management options. A website provides the mechanism by which information and expertise is exchanged between national groups. This paper gives an overview of the approach being adopted, describes initial progress and outlines the expected benefits of such a network.
\end{abstract}

\section{INTRODUCTION}

Widespread contamination of the foodchain following a nuclear accident could have considerable consequences for European farming and food industries. For the purposes of contingency planning it is important to bring together the many and diverse stakeholders who would be involved in intervention so that strategies can be developed for maintaining agricultural production and food safety. This type of approach has been successfully implemented in the UK through the setting up of the Agriculture and Food Countermeasures Working Group in 1997 [1,2]. However, accidental releases of radioactivity do not respect national boundaries and subsequent contamination of the foodchain could affect several Member States. The BSE crisis, problems with dioxin contaminated animal feed and more recently Foot and Mouth disease have highlighted the need for good lines of communication between countries. With financial support from the European Commission (EC), the FARMING network of stakeholder organisations is being set up, initially comprising groups from the UK, France, Finland, Belgium and Greece. The four-year project, which started in November 2000 , will evaluate the practicability of a wide range of management options under the diverse range of food production systems encompassed by the participating Member States. This paper gives an overview of the approach being adopted, describes initial progress and outlines the expected benefits of such a network.

\section{OBJECTIVES}

The overall objective of the project is to set up a network of stakeholder working groups in 5 Member States, involving more than 50 individual stakeholders, to assist in the development of robust and practicable strategies for restoring and managing contaminated agricultural land and food products in a sustainable way. Specific objectives are:

- To identify within each participating Member State the principal stakeholder organisations that would be affected by contamination of agricultural land and food products

- To set up national working groups of these stakeholder organisations and to network them via a web site 
- To identify topics for discussion by the groups, prepare the relevant discussion papers, organise annual meetings and feedback information from national meetings to the other networked groups

- To organise a workshop for participating as well as non-participating Member States with a view to future extension of the network

\section{APPROACH}

An overview of the approach being taken in the FARMING project is given in Figure 1. Experience gained in the UK from the setting up and operation of the Agriculture and Food Countermeasures Working Group is being fed into a steering group, which is responsible for overseeing the formation of similar stakeholder groups in other Member States. These stakeholder groups will meet annually to discuss restoration strategies. Information and expertise from meetings will be exchanged with other national groups via a web-site, and reconsidered in an iterative process. A series of reports will be produced and web-site articles will consolidate principal findings from a European perspective. A workshop will be organised in the final year of the project to describe state-of-the-art restoration strategies and to advertise the existence of the stakeholder network.

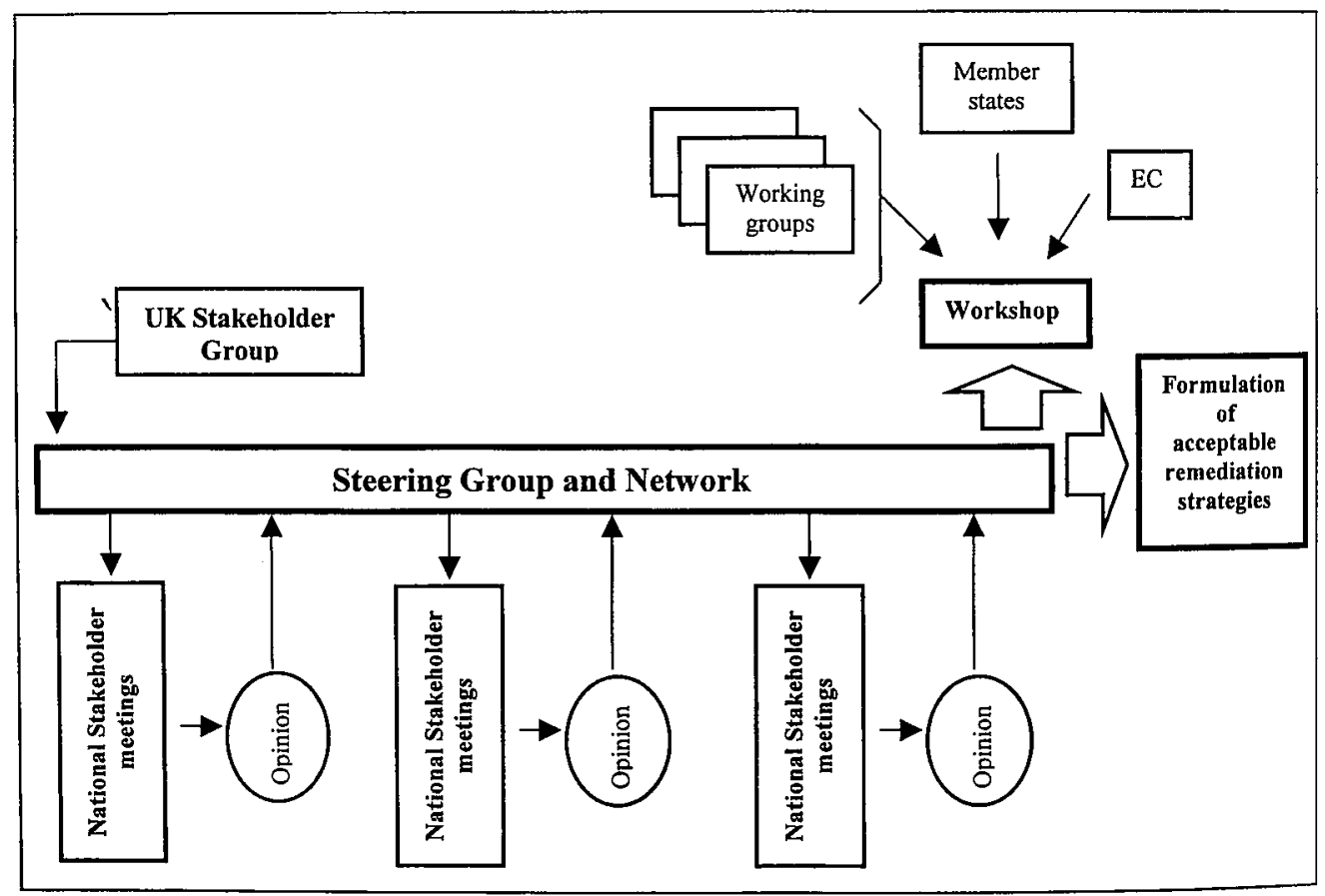

Figure 1: Schematic diagram of FARMING project

\section{PROGRESS}

A report has been produced detailing the operation and achievements of the UK group over the period 1997-2000 [1]. A web-site has also been launched (www.ec-farming.net) to provide a focus for the FARMING network and act as a medium for fast and accessible information exchange. The Steering Group has agreed draft terms of reference for stakeholder groups and discussed potential membership and formats for meetings. The first series of stakeholder group meetings is scheduled to take place in autumin 2001. 


\subsection{Terms of reference}

At their first meeting, stakeholders will be asked to agree terms of reference for their groups that encompass the following three objectives:

- To establish lines of communication between those organisations who, in the event of a nuclear accident, would be involved in decisions on the need for intervention in agricultural systems in the medium to long term, and in their implementation.

- To provide a forum for the dissemination of relevant information on agricultural countermeasures.

- To debate and judge the practicability of various countermeasure options, as part of pre-accident planning, and to distil the implications for government and the agriculture and food industries; and to identify where further work is required.

Additional terms of reference may be added where the membership considers it appropriate to do so. The UK group for example has an additional function:

- To provide the core of a working group that, in the event of an accident affecting agriculture in the UK, could be convened to provide an input to decisions on countermeasure strategy

\subsection{Membership}

The initial membership composition of each national stakeholder group is given in Table 1 . Chairmanship of each group is also indicated.

Table 1: Types of stakeholder organisations represented on national working groups

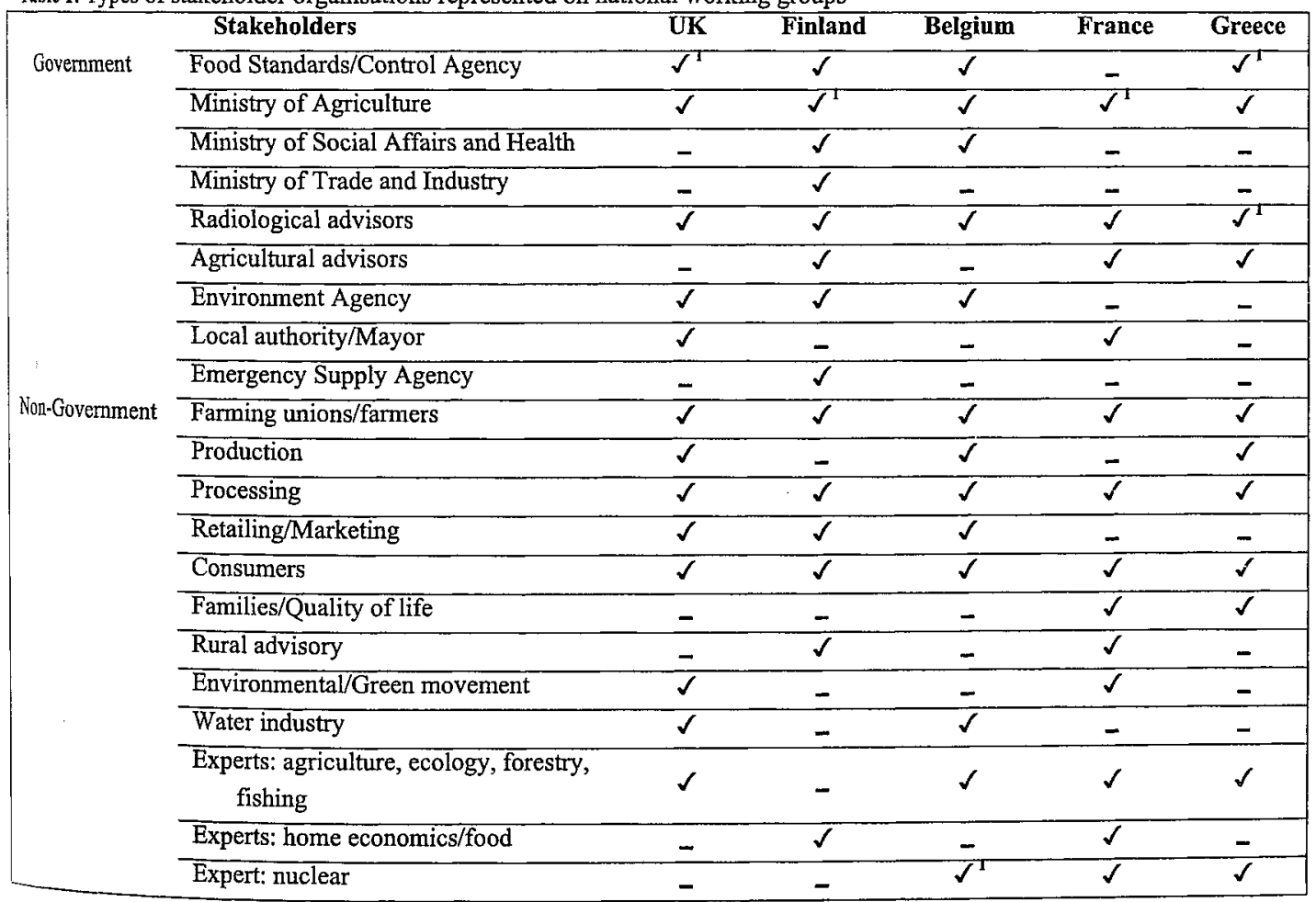

\footnotetext{
${ }^{1}$ Provides chairnerson of national groun
} 
The selection of potential members was based on the following four criteria:

- Adequate representation of the interests and concerns of each stakeholder type.

- Reasonable balance between Government Organisations (GOs) and Non-Government Organisations (NGOs).

- Participation from individuals with responsibility for input to policy type decisions and with a broad knowledge of the issues.

- Willingness to participate.

Key organisations with responsibilities for the food supply chain as well as those affected by the implementation of restoration strategies in rural areas were identified. The diversity of stakeholders selected can be seen in Table 1. In some cases, the same stakeholders are represented in each national group (e.g. representatives from the Ministry of Agriculture, radiological advisers, farming unions and consumers): national differences are also apparent however. Membership will be kept under constant review and evolve as the groups mature. Some of the stakeholders listed are contributing more than one representative either because of the diversity of disciplines of some of the larger organisations or due to regional representation. When this is taken into account, the balance between GOs and NGOs is as shown in Table 2. The high level of involvement of NGOs serves to widen the expertise available and enhance the credibility and independence of the national groups.

Table 2: Representation of GOs and NGOs on national groups

\begin{tabular}{|l|c|c|c|c|c|}
\hline & UK & Finland & Belgium & France & Greece \\
\hline GO & 12 & 9 & 8 & 7 & 8 \\
NGO & 10 & 6 & 13 & 11 & 9 \\
\hline
\end{tabular}

Individuals were selected on the basis of their senior position within their organisations so that authoritative and representative views on a wide range of issues could be presented and discussed at meetings. The Food Standards Agency, Ministry of Agriculture and/or an authority on radiological protection matters have agreed to chair the groups. Partners of the FARMING project will provide the technical secretariat support.

\subsection{Meetings}

The groups will convene annually in the autumn of 2001, 2002 and 2003 for a series of two-day meetings. Additional meetings may also be set up on an ad hoc basis as and when required. The aim of the first meeting is to:

- clarify the stakeholders main concerns following a nuclear accident

- provide an overview of all the underlying issues

- provide an overview of countermeasure options for restoring food production

- prepare for a scenario

Background information on nuclear emergency arrangements, radionuclide transfer in foodchains, and countermeasure strategies will be provided to stakeholders at the outset to promote informed debate. Subsequently, realistic accident scenarios involving contamination of milk, crops, or other important foodstuffs will be used to focus discussion and to elicit stakeholder opinion on a range of management options. In the UK, contamination of milk was found to give members most cause for concern because of the rapid transfer of several important radionuclides from pasture to milk and the continuous nature of its production. Constructive and fruitful discussions within the UK group have enabled new approaches to the minimisation of waste milk arisings and the disposal of waste milk to be developed. A database, containing technical, social and ethical information on these and other state-of-the-art countermeasures 
for rural and semi-natural ecosystems is being constructed in a related EC project (STRATEGY ${ }^{1}$ ). This database will provide a focus for discussions at future meetings of the stakeholder groups and be a useful reference source.

\section{EXPECTED BENEFITS OF THE NETWORK}

\subsection{Improved communication and debate}

The FARMING network will establish lines of communication between those organisations that have not hitherto collectively considered the implications of contamination of the foodchain. The practicability of restoration strategies will be debated at a senior level and in a European context, taking into account regulatory views, social and political factors and industrial constraints. This unique source of information will promote the level of authoritative advice given to Government ministers at the national level and enhance the credibility of information communicated to members of the public. Good communication links at the early stages of an accident will also enable more timely strategic decisions to be made, thereby minimising disruption to the food supply chain. This should help to maintain public's confidence in the authorities managing the accident situation.

\subsection{Wider dissemination of information on restoration strategies}

The network will provide a forum for the dissemination of relevant and up to date information on restoration strategies in advance of a nuclear accident. The FARMING web-site will enable information on practicable as well as impracticable strategies to be exchanged between Member States in a fast and accessible form, thereby avoiding duplication in research effort and the implementation of inappropriate techniques. The final-year workshop will also serve to promulgate information on management strategies and act as a platform for further expansion of the FARMING network in Europe.

\subsection{Applicability to non-nuclear contaminants}

The setting up of a network of stakeholder groups with interests in agriculture and the food industry will ensure that an infrastructure is in place that can potentially deal with other types of contamination event. Some of the stakeholders involved could be the same, as could some of the strategies. The network can therefore be exploited for other purposes in the future.

\section{Acknowledgements}

The author acknowledges the support of her FARMING partners: Aino Rantavaara and Riitta Hanninen (Radiation and Nuclear Safety Authority, Finland); Christian Vandecasteele (Centre d'Etude de l'Energie Nucléaire, Belgium) and Lionel Sombré (Agence Fédérale de Contrôle Nucléaire, Belgium); Henry 0llagnon and Vincent Pupin (Institut National Agronomique Paris-Grignon, France); Kostas Ioannides (University of Ioannina, Greece) and Chryso Tzialla (National Agricultural Research Foundation, Greece). The work is funded by the European Commission Fifth Framework Programme (Nuclear Fission, Radiation Protection) under Contract No: FIKR-CT-2000-20064. The information provided is the sole responsibility of the authors and does not reflect the Commission's opinion. Furthermore, the Commission is not responsible for any use that might be made of the data appearing in this paper.

Sustainable restoration and long-term management of contaminated rural, urban and industrial ecosystems (STRATEGY) a project co-ordinated by BJ Howard and funded by the EC $5^{\text {th }}$ Framework Programme. 


\section{References}

[1] Nisbet A.F. and Mondon K.J., NRPB-R331 (2001)

[2] Nisbet A.F. and Mondon K.J., Stakeholder pre-involvement in the post accident management of nura] areas. OECD/NEA/CRPPH Workshop on Better Integration of Radiological Protection in Modem Society, Villigen, Switzerland 23-25 January 2001 (in press). 